Service social

\title{
Familles recomposées : qu'avons-nous appris au fil des ans?
}

\section{Marie-Christine Saint-Jacques}

Volume 39, numéro 3, 1990

Familles recomposées après divorce

URI : https://id.erudit.org/iderudit/706498ar

DOI : https://doi.org/10.7202/706498ar

Aller au sommaire du numéro

Éditeur(s)

École de service social de l'Université Laval

ISSN

1708-1734 (numérique)

Découvrir la revue

Citer cet article

Saint-Jacques, M.-C. (1990). Familles recomposées : qu'avons-nous appris au fil des ans? Service social, 39(3), 7-37. https://doi.org/10.7202/706498ar

\section{Résumé de l'article}

La croissance importante du nombre de familles recomposées a amené les chercheurs à examiner de plus près cette nouvelle réalité familiale. Cet article tente de cerner l'état des connaissances actuelles dans ce domaine en présentant les résultats de plusieurs études empiriques. Les thèmes ayant le plus retenu l'attention des chercheurs sont abordés dans cette recension des écrits, dont les caractéristiques structurelles de ces familles, la satisfaction et l'ajustement des adultes, le rôle de beau-parent et les effets de la recomposition familiale sur les comportements, les attitudes et les sentiments des enfants. L'auteure tente finalement de faire ressortir de l'ensemble de ces résultats certains constats qu'il serait intéressant d'approfondir dans les recherches futures. 


\section{ARTICLES}

Marie-Christine Saint-Jacques, professionnelle de recherche à l'École de service social de l'Université Laval.

\section{Familles recomposées : qu'avons-nous appris au fil des ans ?'}

Marie-Christine Saint-Jacques

\section{Introduction}

Au cours des vingt dernières années, nous avons assisté au Québec à une transformation importante des modèles de vie familiale. Cette transformation s'est d'abord manifestée par une augmentation sensible du nombre de familles monoparentales et, plus récemment, par l'apparition d'un nouveau type de famille : les familles recomposées. II importe de souligner que toutes les familles recomposées ne sont pas un produit du vingtième siècle. Les familles recomposées d'hier se caractérisaient par le décès du précédent conjoint. Ce qui confère aujourd'hui à la famille recomposée son caractère nouveau, $c^{\prime}$ est qu'elle naît à la suite de la séparation ou du divorce des parents de la précédente famille.

Cette réalité nouvelle a donné lieu à toute une série d'essais et de travaux scientifiques sur le sujet (Clingempeel et al., 1984, Furstenberg et Spanier, 1984; Stollman, 1985; Germain, 1986; Hetherington et Arasteh, 1988; Hutchinson et al., 1989; Boisgelot, 1989; Pasley et Ihinger-Tallman, 1989; Glick, 1989). Une étude empirique portant sur 
le contenu de la littérature populaire (Pasley et Ihinger-Tallman, 1985) a même démontré que ce type d'écrits accordait un intérêt croissant $\mathrm{au}$ thème du remariage et à la situation des personnes vivant avec des enfants nés d'une précédente union. Aux États-Unis, il existe deux associations nationales de familles recomposées. Par ailleurs, au Québec, le phénomène semble suffisamment important et complexe pour que soient mis sur pied, dans différents CLSC, des groupes d'entraide destinés à venir en aide à ces familles. De plus, dans les derniers congrès québécois (dont "La famille, quelles familles ? "2) portant sur la famille, un certain nombre d'ateliers leur ont été consacrés.

Afin de rendre compte de l'état actuel des connaissances sur les familles recomposées, nous avons d'abord examiné les thèmes qui ont le plus retenu l'attention des chercheurs. Ces thèmes sont au nombre de $\operatorname{sept}^{3}: 1$. l'émergence d'une nouvelle réalité familiale, 2. les types de familles recomposées, 3. la spécificité et la complexité des familles recomposées, 4 . une structure familiale anomique, 5. la satisfaction conjugale et l'ajustement chez les familles recomposées, 6 . le rôle de beau-parent et 7 . I'effet de la recomposition familiale sur les enfants. Notons que cet examen des écrits s'est surtout concentré sur les travaux empiriques ${ }^{4}$.

\section{Émergence d'une nouvelle réalité familiale}

Malgré l'intérêt grandissant que l'on observe à l'endroit des familles recomposées, celles-ci ne demeurent pas moins une structure familiale que l'on connaît mal. D'ailleurs, le principal témoin de cette non-connaissance est la langue française. C'est en effet à cet aspect que se heurte en tout premier lieu quiconque s'intéresse au sujet. Si les Américains ont la notion de " step " pour désigner les liens (autres que ceux du sang) qui unissent les membres d'une famille recomposée, nous devons, quant à nous, nous contenter de préfixes (beaupère, belle-mère, beaux-enfants) n'arrivant même pas ainsi à établir la distinction entre les parents des conjoints et la personne qui vit avec des enfants nés d'un autre lit. Pour Théry (1987), cela n'est que le reflet de l'ensemble des incertitudes qui entourent ce type de familles :

De périphrases en guillemets, le langage commun " accroche » aussi bien sur les mots disponibles que sur l'absence de ceux qui s'obstinent à ne pas émerger, comme si l'identification non seulement des relations mutuelles et des statuts respectifs mais, au-delà, des configurations familiales dans lesquelles ils s'inscrivent, restait en quelque sorte toujours inachevée et comme suspendue. (p. 120) 
Par ailleurs, ce type de famille semble entraîner des difficultés spécifiques dont on connaît mal les contours et les facteurs associés. Knaub et Hanna (1984-a) soulignent d'ailleurs que les études menées à ce jour ont souvent cherché à décrire, à définir et à analyser les conflits et les problèmes inhérents à la vie dans une famille recomposée. De plus, ces auteurs ajoutent qu'il y a : "One theme predominant in the remarried family literature; those embarking this family should be prepared for problems »(p. 42).

La recension des écrits que nous avons effectuée leur donne en partie raison. Il faut cependant souligner que les études dans ce domaine ont beaucoup plus cherché à comparer la famille recomposée aux autres structures familiales et à y faire ressortir les sources de stress qu'à explorer les facteurs liés à la qualité de la vie dans ce type de famille (Nolan et al., 1984). Il s'agit d'une façon de faire très populaire dans l'étude de la famille et qui repose, implicitement, sur le principe que la famille "nucléaire ${ }^{5}$ » est le système par excellence permettant un développement maximal et harmonieux de la personne. Aussi, tout autre type de familles, notamment monoparentales ou recomposées, est considéré comme répondant de manière incomplète aux besoins de ses membres et, de ce fait, entraîne des difficultés particulières. Les récents travaux de Coleman et Ganong (1987) abondent dans le même sens en faisant notamment ressortir que toutes les structures familiales "non nucléaires "sont étudiées par comparaison avec la famille nucléaire qui représente le "standard normal ».

Plusieurs des chercheurs (Furstenberg et Spanier, 1984-a; Anderson et White, 1986; Clingempeel et Segal, 1986) qui se sont penchés sur le thème des familles recomposées ont par ailleurs fait état du peu de recherches menées dans ce domaine, alors que " the stepfamily has emerged in large enough numbers to be considered one of the several family types in contemporary social structure " (Jacobson, 1980 : 2). II faut toutefois noter que cette lacune tend à se combler progressivement, puisqu'on assiste actuellement à un foisonnement de recherches s'intéressant aux familles recomposées. On peut se demander si cet intérêt soudain pour la famille recomposée est vraiment le reflet d'une croissance marquée de cette structure familiale. Une façon de répondre à cette question consiste à examiner les statistiques disponibles sur le sujet.

\section{Prévalence des familles recomposées}

Le recensement de 1986 nous indique qu'environ un mariage sur trois se solde par un divorce au Canada. Ces divorces touchent près d'une fois sur deux des enfants à charge (Statistique Canada, 1989). 
Parallèlement, Statistique Canada (1988) évalue que $76 \%$ des hommes et $64 \%$ des femmes divorcés se remarient. II faut aussi noter que de plus en plus de personnes célibataires ou divorcées choisissent de vivre en union de fait (Peron et al., 1987). Une recherche (SaintJacques, en cours) menée auprès de 162 personnes vivant en famille recomposée a fait ressortir que seulement $19,8 \%$ d'entre elles ont choisi de légaliser leur union.

Bien que nous ne possédions que peu de chiffres portant spécifiquement sur la recomposition familiale, l'Enquête sur la famille (Burch, 1985) a permis d'établir qu'au Canada 4,4\% des hommes ont déjà élevé des enfants d'un autre lit. Ce pourcentage est beaucoup plus faible chez les femmes $(2,1 \%)$ reflétant le fait qu'en majorité, ce sont ces dernières qui, à la suite d'un divorce, ont la garde des enfants (Burch, 1985).

Par ailleurs, les chiffres avancés pour les États-Unis sont beaucoup plus élevés. Ceci s'explique notamment par les taux de mariages, de divorces et de remariages de personnes divorcées qui sont plus élevés qu'au Canada (Statistique Canada, 1988). Toutefois, il nous apparaît pertinent de présenter les tendances observées aux ÉtatsUnis, d'une part, parce que la prévalence de la recomposition familiale a amené les Américains à recueillir des statistiques précises sur ce sujet et que, d'autre part, les tendances observées chez nos voisins nous permettent d'envisager, dans une certaine mesure, les tendances futures au Canada.

Ainsi on évaluait en 1984 que $50 \%$ des mariages actuels finiraient par un divorce et que $60 \%$ des personnes divorcées auraient des enfants âgés de moins de 18 ans au moment du divorce (Clingempeel et al., 1984-a). Glick et Lin (1986) ont par ailleurs observé que $68 \%$ des femmes qui se remariaient en 1980 avaient au moins un enfant né d'une union antérieure. On estime actuellement à 4,3 millions le nombre de familles recomposées aux États-Unis, soit $6,7 \%$ de toutes les familles américaines. Une projection statistique permet de prédire que $61 \%$ des hommes vivant en familles recomposées et $54 \%$ des femmes divorceront de nouveau (Glick, 1984, cité dans Pasley et Ihinger-Tallman, 1989).

Si l'on examine la situation des enfants, on constate que $14 \%$ de tous les enfants américains vivent en famille recomposée et que 9,4\% de ces enfants ont un statut de bel-enfant dans leur famille ${ }^{6}$ (Glick, 1989). Finalement, une extrapolation statistique permet d'affirmer que $28 \%$ des enfants vivant actuellement en famille recomposée vivront la séparation de cette seconde famille (Bumpas, 1984, cité dans Brody et al., 1988).

La lecture de ces statistiques nous indique donc que le nombre de familles recomposées est en progression. 


\section{Les types de familles recomposées}

Pour Germain (1986), il existe trois types de familles recomposées. Cette typologie se fonde d'abord sur les motifs de recomposition, soit le divorce ou le veuvage. Par ailleurs, à l'intérieur des familles recomposées à la suite d'un divorce, elle établit deux autres types, selon que le parent a une garde occasionnelle ou permanente des enfants issus de sa précédente union.

Knaub et Hanna (1984-a) ont pour leur part retenu, dans le cadre de leurs travaux sur les familles recomposées, la définition suivante : au moins un des deux conjoints a déjà été marié et est divorcé, au moins un enfant d'une union antérieure vit avec le couple de façon permanente. II y a présence d'un autre parent qui n'a pas la garde. Cette définition n'englobe donc pas les familles recomposées à la suite d'un veuvage ou n'ayant qu'une garde occasionnelle de l'enfant.

Visher et Visher (1979) utilisent pour leur part le terme famille recomposée pour inclure tous les systèmes familiaux dans lesquels au moins un des deux conjoints est un beau-parent et où au moins un des deux conjoints a un enfant d'un mariage précédent. L'enfant peut faire des visites au couple ou demeurer avec lui.

Clingempeel et al. (1984-b) ont par ailleurs nuancé le type de famille recomposée, selon qu'un seul des deux conjoints a des enfants nés d'une précédente union (famille recomposée simple) ou que les deux conjoints ont des enfants nés d'une précédente union (famille recomposée complexe).

Finalement, une typologie à laquelle font de plus en plus référence les chercheurs pour étudier la famille recomposée est celle qui consiste à subdiviser cette dernière selon qu'elle compte une bellemère ou un beau-père. Nous verrons au cours de cette recension toute l'importance de cette division.

\section{Spécificité et complexité des familles recomposées}

Lorsqu'on examine les qualificatifs donnés par les chercheurs (Goldstein, 1974; Duberman, 1973; Cherlin, 1978; Goetting, 1982) à la famille recomposée, on observe qu'il est fréquent que ces derniers la définissent comme une structure familiale complexe. Un premier élément justifiant cette complexité vient du fait que ce type de familles met en relation un grand nombre de personnes. À ce sujet, Visher et Visher (cités dans Cuerrier, 1982) ont fait une éloquente démonstration mathématique du nombre de paires et d'interactions possibles au sein d'une famille recomposée. Alors que dans une famille nucléaire comprenant un père, une mère, leurs parents respectifs et 
deux enfants le nombre de paires s'élève à 28 et les interactions possibles à 247 , ces chiffres passent respectivement à 253 paires et au nombre faramineux de 8388584 interactions possibles dans une double recomposition (famille recomposée complexe). II a suffi pour cela que le couple se sépare et se remarie, madame avec un divorcé père de trois enfants et monsieur avec une veuve mère d'un enfant. Le très grand nombre de personnes touchées est donc pour nous une première caractéristique structurelle qui distingue les familles recomposées des familles nucléaires et qui vient expliquer du même coup la complexité de la vie au sein de ce type de famille.

Un autre élément distinctif a trait au processus traditionnel du cycle de la vie familiale. Ce cycle conçoit que la famille nucléaire se forme à partir de l'union de deux adultes qui auront ensemble des enfants. Dans les familles recomposées, il y a au moins un lien parentenfant qui existe avant les liens du couple (Visher et Visher, 1990). Dans un même ordre d'idée, tous les individus qui s'unissent dans ce type de famille ont déjà une histoire familiale (Papernow, 1984; Visher et Visher, 1990) ${ }^{7}$. De ce fait, les valeurs et les styles de vie des adultes et des enfants peuvent être très différents et parfois conflictuels.

Par ailleurs, les familles recomposées, contrairement aux familles nucléaires, naissent à partir de pertes relationnelles importantes pour ses membres, telles que le divorce, ou la perte du rêve de ce que leur mariage aurait pu être (Visher et Visher, 1989). Pour le conjoint qui en est à sa seconde union, la perte relationnelle que représente le divorce peut ne pas être résolue (1979). Ainsi, le deuil de la famille précédente est pour Capaldi et McRae (1979) un élément important de I'adaptation de la nouvelle famille. À ce sujet, Goetting (1982) ajoute que le remariage peut être un processus complexe, d'autant plus complexe que le deuil de la relation précédente n'est peut-être pas réglé :

The problems associated with remarital adjustment are often heightened by the fact that partners in remarriage may still be adjusting to their divorces. At remarriage, a person may be compelled to commence the stations of remarriage while having not yet completed the stations of divorce. (p. 222)

Sur un autre plan, peu de liens légaux existent entre le beauparent et les enfants à moins d'avoir effectué certaines démarches légales. Il faut cependant noter que des démarches légales, comme l'adoption des enfants du conjoint, sont, sauf quelques rares exceptions, pratiquement inexistantes chez les familles recomposées à la suite d'un divorce. Cette absence de liens légaux peut entraîner des situations déchirantes. Ce serait le cas notamment d'un beau-parent qui, après avoir vécu 5,10 ou 15 ans avec un enfant, pourrait ne se voir 
reconnaître aucun droit de visite à cet enfant à la suite du décès du parent naturel et conjoint dans la famille recomposée.

Si l'on se penche spécifiquement sur la question des enfants, on constate que ces derniers peuvent passer du temps dans deux maisons ayant différentes règles et attentes les concernant. Ils peuvent aussi, après la recomposition, voir leur rang dans la famille se modifier ou se retrouver " instantanément " avec des demi-frères et des demi-sœurs. De plus, ils peuvent éprouver des conflits de loyauté, notamment dans leur relation avec leur beau-parent et le parent naturel qui n'a pas la garde.

Sous un angle systémique, ce type de système familial a souvent des frontières plus ouvertes du fait qu'il y a quelque part un exconjoint ou une ex-conjointe parent biologique des enfants (Walker et Messinger, 1979). La coexistence de ces deux familles, c'est-à-dire "I'ancienne et la nouvelle », pourrait, selon Perkins et Kahan (1979), amener une confusion systémique qui de ce fait compromet le contexte relationnel (nous verrons au thème de l'ajustement des enfants que cette caractéristique a effectivement une influence sur les relations familiales). La famille, et plus spécialement le beau-parent, peut percevoir que par les enfants l'ex-conjoint est un intrus dans la famille actuelle (Walker, 1984). De plus, cet ex-conjoint peut, même s'il n'appartient pas au système familial, exercer un pouvoir sur le système du fait de son droit de regard et de sa relation avec ses enfants. Statistiquement cependant, Trost (1984), dans une étude sur le remariage en Suède, a noté que les personnes remariées avaient moins de contacts avec l'ex-conjoint que les personnes séparées ou divorcées.

L'examen de ces caractéristiques structurelles nous amène à conclure qu'une famille recomposée est différente d'une famille nucléaire. Mais le fait d'être différent constitue-t-il en soi un problème ? Des impressions cliniques, de même qu'un certain courant de recherches (défini par Théry [1987] comme les études portant sur la « famille anomique »), nous incitent à croire qu'une des difficultés importantes de la vie en famille recomposée vient en partie du fait qu'elles n'ont pas de modèles, pas de normes sur lesquels s'appuyer et qu'ainsi les rôles de chacun des membres sont pauvrement définis.

\section{Une structure familiale anomique}

En 1966, Fast et Cain soulignaient déjà que la capacité d'un beauparent à assumer son rôle ne dépend pas uniquement de son bon vouloir, mais aussi de l'acceptation réciproque du conjoint et des enfants dans ce rôle. La société ne fournit pas non plus de normes, mais refléterait plutôt certaines idées contradictoires : "The role de- 
finition of stepparent in this society is both poorly articulated and implies contradictory functions as "parent ", "stepparent ", and "nonparent» (p. 486).

D'autres chercheurs (Wilson et al., 1975; Clingempeel et al., 1984-a) ont aussi souligné que l'absence de règles et de rôles bien définis caractérisaient les familles recomposées. Pour Walker et Messinger (1979) cette notion de rôle constitue un élément distinguant les familles recomposées des familles nucléaires. Le concept de rôle est utilisé par ces chercheurs au sens du degré de clarté des rôles et du degré où un rôle est attribué à quelqu'un vs accompli par quelqu'un. Par exemple, si dans une famille nucléaire il va de soi qu'un père s'occupe de ses enfants, dans les familles recomposées "they are simply no clearly defined role prescriptions concerning the rights and obligations between new spouse and partner's children » (p. 187).

Cherlin (1978) ainsi que Furstenberg et Spanier (1984-a) se sont aussi intéressés aux problèmes soulevés par le fait de vivre dans une structure familiale où les normes sociales ne sont pas clairement définies. À ce sujet, Cherlin (1978) dans une étude aujourd'hui très connue ("Remarriage as an Incomplete Institution») a tenté d'expliquer sur le plan théorique le taux supérieur de divorces que l'on retrouve chez les familles recomposées par rapport à celui observé chez les familles nucléaires. Partant du fait que l'unité familiale semble plus difficile à maintenir, Cherlin a posé comme hypothèse que les difficultés vécues par les couples dans les remariages proviennent d'un manque de modèles institutionnalisés pour régler plusieurs problèmes courants de la vie dans ce type de famille. Afin d'étayer cette thèse, Cherlin mentionne que le vocabulaire et la loi (qui sont deux des plus importantes institutions de notre société) ne précisent rien pour les familles recomposées. Cela illustre le fait que le remariage est une institution incomplète. Cherlin soutenait aussi le fait que les secondes unions comprenant des enfants couraient un risque plus élevé de divorce. Quelques années plus tard, Furstenberg et Spanier (1984-a) ont examiné empiriquement l'hypothèse avancée par Cherlin. Les résultats ont permis de confirmer que les familles recomposées font face à des problèmes pour lesquels aucune solution institutionnalisée n'a émergé. Cependant, ces résultats n'ont pas appuyé l'idée que les secondes unions avec des enfants couraient un risque plus grand de séparation ou de divorce. Cette étude préconisait plutôt une autre explication au haut taux de divorce parmi les secondes unions, soit le fait que les personnes divorcées acceptent plus facilement l'idée du divorce comme une solution à un mariage insatisfaisant. 


\section{Conséquences de l'absence de normes sociales}

Pour Giles-Sims (1987), les chercheurs ont peu étudié la façon dont le couple dans la famille recomposée définit les rôles de chacun des partenaires, même si bon nombre d'études considèrent que l'absence de normes institutionnalisées influence la clarté des attentes $\mathrm{d}^{\prime}$ un individu. De plus, il indique que lorsqu'une personne perçoit clairement les attentes que l'on a à son égard, elle performe mieux et retire de ce fait plus de satisfaction relativement aux rôles qu'elle occupe.

De plus, pour Visher et Visher (1979), le caractère invisible des familles recomposées conduit à une méconnaissance de ce type de famille. De cette méconnaissance naît un certain nombre de mythes. Ainsi, tout en étant privée de modèles, la famille recomposée est aux prises avec de fausses croyances auxquelles même ses membres n'échappent pas. Ayant trouvé à plusieurs reprises dans la documentation clinique la mention de ces mythes comme pouvant handicaper le fonctionnement des familles recomposées, nous croyons important de les mentionner ici.

1. Le mythe de la belle-mère cruelle (Visher et Visher, 1978; Capaldi et McRae, 1979; Nelson et Nelson, 1982; Walker, 1984).

2. Les familles recomposées sont comme les familles nucléaires (Visher et Visher, 1978).

3. Le décès du précédent conjoint facilite la recomposition (Visher et Visher, 1978).

4. Les beaux-enfants sont plus faciles à contrôler quand ils ne vivent pas avec le couple (Visher et Visher, 1978; Ambert, 1986).

5. L'amour naît instantanément (Visher et Visher, 1978; Capaldi et McRae, 1979; Nelson et Nelson, 1982).

\section{Satisfaction conjugale et ajustement chez les familles recomposées}

Une famille recomposée peut-elle "réussir »? Une façon de répondre à cette question est d'examiner les taux de satisfaction et les taux de divorce! Nous avons déjà établi que les taux de divorce sont un peu plus élevés chez les familles recomposées que chez les premières unions (de $60 \%$ chez les personnes vivant une seconde union contrairement à $49 \%$ chez les premières unions). Par ailleurs, selon plusieurs études (Albrecht, 1979; Glenn et Weaver, 1977; Demaris, 1984; White et Booth, 1985; Hetherington, 1987), les secondes unions seraient des mariages heureux et aussi satisfaisants qu'un premier 
mariage. Pour Albrecht (1979), ces unions seraient même plus satisfaisantes que les premiers mariages, à tout le moins durant les deux premières années (Hetherington, 1987). Toutefois, Glenn et Weaver (1977) indiquent que les hommes seraient plus satisfaits de leur remariage, alors que les femmes se disent aussi satisfaites par le second mariage que par le premier.

Roberts et Price (1987) ont mené une étude très intéressante sur I'ajustement des couples vivant en famille recomposée. Utilisant une méthode qualitative, ils ont recueilli leur matériel à l'aide d'entrevues en profondeur auprès de seize couples. Les femmes composant cet échantillon avaient des enfants nés d'une précédente union, alors que les hommes en étaient à leur première union. Les résultats ont fait ressortir que les principales attentes des femmes face au remariage se situaient autour des notions de liberté, d'indépendance et d'une bonne communication avec le conjoint. Au chapitre des responsabilités et des rôles, les femmes estiment que le remariage se caractérise par l'expérience de la conjointe comparativement à l'inexpérience de l'homme. La femme se placerait en enseignante visà-vis de son conjoint, cherchant à donner le ton à ce que sera la famille. L'homme serait perçu comme voulant " mettre de l'ordre " dans le fonctionnement familial. Les conjointes jugent les hommes très rigides sur ce point et considèrent qu'ils veulent prendre trop de responsabilités.

Les hommes, de leur côté, cherchent à établir des relations complémentaires avec leur conjointe. Leurs attentes face à la nouvelle famille portent surtout sur le développement d'une sécurité émotive et financière. Par ailleurs, ils perçoivent leur conjointe comme une femme indépendante et forte. Pour ces hommes, leur nouvelle famille correspond à la recherche d'un parent sur qui ils peuvent compter, à l'inverse des précédentes relations qu'ils entretenaient et qui se caractérisaient par l'instabilité et le hasard des rencontres. Ces conjoints sont par ailleurs tourmentés par l'attention que la conjointe et les enfants portent au père naturel. Même s'ils sont préoccupés par le fait que leur conjointe reçoit peu ou pas de pension alimentaire de l'ex-conjoint, il encouragent rarement les contacts avec ce dernier.

Par ailleurs, et toujours selon l'étude de Roberts et Price (1987), ces hommes disent subir une grande pression à se conformer au modèle de fonctionnement de la famille qu'ils sont venus rejoindre. Sur le plan de la relation conjugale, ils précisent qu'un des principaux problèmes touche les relations sexuelles. Cette situation s'expliquerait par le très grand soin mis à définir de nouveaux rôles et à performer à l'intérieur de ces rôles, de même que par la culpabilité que ressentirait la conjointe à accorder du temps et de l'importance à des activités se situant dans une sphère où les enfants sont exclus. Géné- 
ralement, chez ces couples, la satisfaction sexuelle augmente au fur et à mesure que les conflits parentaux se résolvent et que se développe une plus grande expression verbale des sentiments entre conjoints. Sur un autre plan, les beaux-pères ont indiqué que l'arrivée d'un bébé issu de la nouvelle famille renforce et solidifie l'unité familiale, notamment en légitimant le rôle de parent du beau-père. Finalement, les chercheurs ont constaté chez ces beaux-pères une volonté tenace à trouver des solutions et à faire des compromis afin que la nouvelle famille réussisse.

\section{Facteurs associés à la satisfaction et à l'ajustement}

Des auteurs ont cherché à décrire certains éléments influençant cette satisfaction. Clingempeel (1981), dans une première étude sur la satisfaction conjugale et le maintien des contacts avec les membres du réseau du premier mariage (quasi-kin), a démontré que les familles recomposées maintenant un niveau de contact modéré avec le quasikin avaient un taux de satisfaction conjugale plus élevé que celles qui ont maintenu un niveau de contact bas ou élevé ${ }^{8}$. Clingempeel et Brand (1985) ont par la suite refait cette étude et en sont arrivés à la conclusion que ce n'étaient pas les contacts avec le quasi-kin qui influençaient le taux de satisfaction des époux, mais bien le fait qu'un seul parent ou les deux aient des enfants. Ces résultats vont un peu dans le sens des conclusions auxquelles en sont arrivés White et Booth (1985). En effet, selon cette étude, les personnes ayant des beaux-enfants ont un taux de satisfaction plus bas. Les enfants seraient un facteur déstabilisant à l'intérieur des remariages et contribueraient à son haut taux de divorce (cependant, rappelons que Furstenberg et Spanier (1984-a) dans une précédente étude en sont arrivés à des résultats différents). Ils ont de plus constaté que le taux de divorce le plus élevé se situait chez les familles recomposées où les deux parents en étaient à une seconde union.

Du côté des variables qui semblent hausser le taux de satisfaction conjugale, le fait d'avoir cohabité ensemble avant le remariage a été défini par Hanna et Knaub (1981) comme étant un facteur positif. L'étude de Pasley et al. (1984) indique que les couples remariés heureux partagent plus souvent les mêmes perceptions sur leur degré d'accord ou de désaccord que les couples remariés malheureux. Enfin, certains travaux ont démontré que les mères remariées avaient, globalement, un sentiment de compétence et de bien-être plus élevé (Mitchell, 1983) et qu'elles étaient plus satisfaites de leur vie, avaient plus de contrôle interne sur le déroulement de leur vie, vivaient moins de solitude et de dépression (Hetherington, 1987) que les mères divorcées. 
Sur le plan de l'ajustement familial, Knaub et Hanna (1984-a) ont cherché à reconnaître les facteurs contribuant le plus à la définition de forces familiales chez les familles recomposées $(N=160)$. Les facteurs relevés ont été : 1 . I'attitude de l'environnement et 2 . le revenu. Le fait de recevoir de l'aide psychosociale après le remariage a quant à lui été associé plus faiblement aux forces familiales.

Anderson et White (1986) ont pour leur part tenté certaines variables qui distingueraient les familles nucléaires des familles recomposées et les familles recomposées fonctionnelles des familles dysfonctionnelles. Les variables mesurées étaient : 1. I'ajustement conjugal, 2 . les liens entre le parent biologique et les enfants, 3 . le fait d'être peu enclin à exclure des membres de la famille et 4 . I'habileté à faire mutuellement des compromis dans les décisions familiales. Les résultats obtenus indiquent que les familles recomposées fonctionnelles sont semblables aux familles nucléaires fonctionnelles, de même que les familles recomposées qui sont dysfonctionnelles le sont pour les mêmes raisons que les familles nucléaires (par exemple, faible coalition parentale, manque d'habileté dans la prise de décision, etc.). De plus, cette étude a fourni une donnée inattendue, à savoir que l'ajustement conjugal dans les familles recomposées dysfonctionnelles était meilleur que celui mesuré chez les familles nucléaires dysfonctionnelles.

Finalement, la question des frontières semble avoir un effet sur l'ajustement des familles recomposées. Ce dernier point a par ailleurs été abordé par Pasley et Ihinger-Tallman (1989) dans une étude portant sur l'ambiguïté des frontières dans les familles recomposées. Cette recherche, menée auprès de 216 couples, a fait ressortir que certains types de familles recomposées semblent vivre plus de problèmes de frontières que d'autres. Notons que la notion d'ambiguïté des frontières renvoie ici aux concepts de frontières physiques (qui vit avec qui) et psychologiques (qui est considéré comme faisant partie de la famille). Ainsi, dans cette étude, les familles recomposées avec belle-mère semblaient plus susceptibles de vivre ce type de problème, notamment chez les familles où la conjointe vit de façon permanente avec ses enfants, alors que les enfants du conjoint ne viennent qu'occasionnellement. Par ailleurs, cette étude a aussi démontré que le taux d'ambiguïté des frontières n'affecte pas le degré d'ajustement conjugal et d'intégration.

Jusqu'à maintenant nous nous sommes attachés à certains éléments problématiques liés à la famille recomposée. Nous explorerons maintenant les difficultés spécifiquement liées au rôle de beauparent. 


\section{Le rôle de beau-parent}

À la lumière de la théorie de l'échange social, Nelson et Nelson (1982) ont relevé différents problèmes vécus soit par la belle-mère, soit par le beau-père. Les mythes que nous avons présentés précédemment, et plus particulièrement celui de la belle-mère cruelle et de l'amour instantané (afin de lutter contre le précédent mythe), seraient les problèmes les plus importants des belles-mères (Visher et Visher, 1978; Nelson et Nelson, 1982; Walker, 1984). Il est par ailleurs intéressant de noter que ce mythe semble plus persistant chez les bellesmères que chez les enfants. En effet, Dukes (1989) a examiné l'évaluation que faisaient des adolescents du statut de beau-parent. Les résultats recueillis $n^{\prime}$ ont fait ressortir aucune différence significative dans l'évaluation que faisaient ces derniers de l'efficacité des parents par rapport à celle des beaux-parents ou des enfants naturels comparativement aux beaux-enfants par rapport à différentes situations d'interaction familiale. Par ailleurs, Coleman et Ganong (1987) ont conclu de leur recension des écrits que, de façon générale, la société entretient moins de stéréotypes positifs à l'endroit de la famille recomposée qu'à l'égard de la famille nucléaire.

Si l'on revient plus précisément au rôle de beau-parent, on constate que les belles-mères ont tendance à entretenir des attentes irréalistes à l'endroit de la famille, attentes souvent fondées sur le modèle de la famille nucléaire (Duberman, 1975; Visher et Visher, 1979). Par ailleurs, l'expérience d'être belle-mère s'avère plus positive lorsque les enfants vivent en permanence avec la famille et non uniquement en visite les fins de semaine par exemple (Ambert, 1986). Finalement, d'autres difficultés liées à la position de belle-mère apparaissent lorsque l'on examine différents aspects de la relation belle-mère-enfant. Aussi reviendrons-nous sur cette question un peu plus loin.

Pour le beau-père les principales difficultés se feraient sentir sur le plan de la discipline, de la culpabilité, de la loyauté et de l'argent. Pour Visher et Visher (1978) et Hetherington (1987) la discipline deviendrait particulièrement problématique pour le beau-père quand ce dernier cherche à avoir de l'autorité auprès d'enfants avec qui il n'a entretenu qu'une courte et petite relation. Ces problèmes peuvent aussi survenir quand il doit vivre avec l'ambivalence de la mère qui veut du soutien de son conjoint en matière d'éducation, mais qui cherche en même temps à protéger ses enfants.

Ainsi, selon Capaldi et McRae (1979), deux dangers guettent le beau-père : celui de penser qu'il va savoir instantanément quoi faire ou, à l'opposé, celui de décider de ne pas s'impliquer du tout et de laisser le parent naturel tout assumer. Ces problèmes résulteraient généralement d'une non-clarification des rôles dans le couple. Par 
ailleurs, il semble que la qualité de la relation entre les enfants et le beau-père soit modulée par l'attitude de ce dernier. En effet, et contrairement à ce que l'on observe chez les familles nucléaires, les enfants manifesteraient une attitude de rejet et des problèmes de comportement face à un beau-père qui cherche à exercer un contrôle sur eux. La même tendance s'observe lorsque l'attitude du beau-père est une attitude de désengagement total vis-à-vis des enfants dès le début du remariage (Hetherington, 1987).

Toujours sur le plan de l'attitude du beau-père, Hetherington (1987) a noté que les éléments facilitant le plus l'adaptation des garçons dans la famille recomposée sont l'adoption d'une attitude chaleureuse avec ces derniers et le soutien à la mère. Cette façon d'agir permettrait au beau-père d'en venir progressivement à occuper un rôle d'autorité parentale. Cependant, cette même attitude n'aura pas le même impact auprès des filles qui n'accepteront et ne considéreront que très rarement le beau-père dans une position d'autorité. Notons que dans cette recherche, le désengagement parental était le rôle le plus souvent adopté par les beaux-pères et que cette attitude s'accentuait avec le temps, spécialement dans la relation avec les filles de la conjointe.

Sur un autre plan, on constate que le beau-père peut se sentir en conflit de loyauté ou ressentir de la culpabilité notamment en s'occupant des enfants de sa conjointe alors qu'il a déjà des enfants d'une précédente union qui, dans la majorité des cas, ne vivent pas avec lui (Visher et Visher, 1978). Toutefois, il apparait que la relation beaupère-garçon n'est pas affectée par le fait que chacun des parents ait ou n'ait pas d'enfants d'un premier mariage, ni par le sexe de l'enfant (Clingempeel et al., 1984-b). De façon générale, on constate cependant que le rôle de beau-père pose moins de problèmes que celui de la belle-mère. Selon Visher et Visher (1978), cette situation s'explique par le fait que les hommes s'impliquent traditionnellement moins auprès des enfants, qu'il y a moins d'attentes en ce qui les concerne et, de ce fait, moins de tension.

Par ailleurs, la recherche d'Amato (1987) portant sur l'adaptation et le développement des enfants dans les familles nucléaire, monoparentale avec mère chef de famille et recomposée a fait ressortir que les beaux-pères fournissent moins de soutien, et qu'ils contrôlent et punissent moins que les pères biologiques en famille nucléaire. Cependant, l'engagement du beau-père auprès des enfants tend à augmenter au fur et à mesure de la durée de la recomposition. Ce dernier point va cependant à l'encontre des résultats obtenus par Hetherington (1987).

Mentionnons, en terminant, que la relation beau-parent-enfants semble influencée par le sexe de l'enfant. En effet, la relation qu'entretient un beau-parent avec les filles de son conjoint ou de sa conjointe serait teintée de plus de détachement et de moins d'amour. La 
mesure des comportements a révélé que les filles exprimaient moins de messages verbaux positifs et avaient plus de comportements de résolution de problèmes négatifs que les garçons envers leurs beauxparents. Ces derniers, pour leur part, ne diffèrent pas dans leurs réponses aux garçons et aux filles (Clingempeel et al., 1984-a).

\section{Effets de la recomposition familiale sur les enfants}

Tout un courant de recherches s'est intéressé à l'effet de la recomposition familiale sur les enfants. Différentes dimensions ont été examinées, dont les effets de la recomposition sur l'adaptation, la conception de soi et la santé physique et psychologique des enfants.

Dans une recension des écrits portant sur les enfants vivant en famille recomposée, Ganong et Coleman (1987) ont examiné et comparé les résultats obtenus dans les recherches empiriques et les travaux cliniques. De cette comparaison, il ressort peu de congruence entre les résultats des chercheurs et ceux des cliniciens. En effet, alors que la recherche empirique trouve généralement peu de différences entre les beaux-enfants et les enfants vivant en famille nucléaire, les études cliniques en arrivent à la conclusion que les familles recomposées ont des difficultés inhérentes à cette structure familiale et que de ce fait les enfants ont des problèmes d'adaptation.

Les résultats de cette analyse soulèvent à notre avis un problème méthodologique lié au type de population étudiée dans les recherches portant sur la recomposition. En effet, il est probable que les recherches menées par les cliniciens soient davantage centrées sur les individus ayant recours à leurs services. Il devient donc prévisible que les difficultés rencontrées par ces personnes soient plus élevées que celles que l'on retrouve dans une population dite normale.

Par ailleurs, la recherche dans le domaine de la recomposition ne jouissant pas d'une longue tradition, il est fort probable qu'un certain nombre de facteurs associés à l'ajustement des enfants n'aient pas encore été relevés. Ces constatations expliquent, selon nous, certaines des contradictions qui ressortent des thèmes qui suivent.

\section{Ajustement des enfants}

De façon générale, on évalue que les enfants profitent avec avantage du fait de vivre en famille recomposée plutôt qu'en famille monoparentale. À long terme, la recomposition familiale ne semble pas entraîner de différences significatives sur le plan des caractéristiques sociales et psychosociales des adultes selon qu'ils ont été élevés, 
lorsqu'ils étaient enfants, dans une famille nucléaire ou dans une famille recomposée (Wilson, 1975; Bachrach, 1983).

Le degré d'ajustement des enfants semble cependant varier selon le sexe. On observe en effet que les garçons s'adaptent mieux à une situation de recomposition que les filles (Hetherington et al., 1985; Mitchell, 1983; Bray, 1988). Cette meilleure adaptation se manifesterait entre autres par une augmentation de la performance intellectuelle et une diminution du stress. Les filles de leur côté ressentent plus de stress, ont une performance intellectuelle plus faible et manifestent plus de problèmes de comportement que les enfants de famille nucléaire (Bray, 1988).

Outre le sexe de l'enfant, différents facteurs semblent expliquer le degré d'adaptation des enfants. Ainsi certains éléments propres à la dynamique familiale, dont la cohésion et l'engagement émotif, influencent ce degré d'adaptation (Bray, 1988; Pasley et Healow, 1988). Mais même en tenant compte de ces facteurs, le sexe de l'enfant demeure une variable importante. Ainsi les filles ont un niveau d'ajustement plus élevé lorsque les liens affectifs et émotionnels sont moins forts avec leur mère gardienne, alors que les garçons profitent d'une plus grande cohésion et de liens affectifs plus forts avec leur mère et avec leur beau-père.

II faut cependant nuancer ces résultats. En effet, les travaux de Santrock (1982) portant sur les comportements sociaux des enfants et des parents dans les familles recomposées avaient amené l'auteur à conclure que :

the social behavior of children is not necessarily less competent in stepfather families that in intact or divorced families. The data suggested that such factors as parenting behavior, sex of child, and marital conflict in any type of family structure are implicated as possible explanations of the child's social behavior. (p. 242)

Ainsi, bien que les variables de sexe et de comportement des enfants soient importantes, elles n'ont pas une portée différente, selon Santrock (1982), parce que l'on vit en famille recomposée. Ce constat rejoint par ailleurs les résultats plus récents de Kurdek et Sinclair (1988). En effet, la comparaison qu'ils ont effectuée entre des adolescents de familles nucléaire, monoparentale et recomposée a démontré que ni la structure familiale ni le sexe de l'adolescent ni son degré scolaire n'ont d'influence sur son ajustement. Tout comme dans l'étude de Santrock (1982), l'ajustement a été trouvé inversement associé au niveau de conflits familiaux. Pour les trois structures familiales examinées, un ajustement positif était associé au soutien social à l'extérieur de la famille et à l'utilisation de stratégies positives d'adaptation (comme être engagé dans des activités de loisirs, se confier à des amis, etc.). 
II ressort aussi des travaux de Clingempeel et Segal (1986) que la qualité de la relation belle-mère-enfants a une influence sur l'ajustement de ces derniers. En effet, dans cette étude les chercheurs ont remarqué qu'une relation plus positive entre la belle-mère et les enfants était liée à un bas taux d'inhibition et d'agressivité chez les garçons et les filles et à un haut niveau d'estime de soi chez les filles. La durée de la recomposition semble aussi influencer la qualité de la relation belle-mère-fille : plus la recomposition date, meilleure est la relation et plus bas sont les taux d'agressivité et d'inhibition chez les filles. Peu de différences significatives ont été observées chez les enfants vivant avec un beau-père.

La performance et le développement cognitif des enfants sont un indicateur souvent considéré dans l'évaluation de l'ajustement des enfants. Chapman (1977) a donc utilisé cet indicateur afin de comparer l'ajustement d'adolescents vivant dans différents types de familles. Les résultats suggèrent qu'un beau-père peut agir comme un remplaçant du père naturel dans le développement cognitif de l'enfant. L'absence du père aurait été associée à un haut taux de dépendance chez l'enfant et à un bas taux d'aptitudes scolaires. La présence d'un beau-père aurait tendance à atténuer ce phénomène.

Si la présence d'un beau-père semble compenser l'absence du père, il n'en va pas de même lorsqu'il s'agit de famille recomposée comprenant une belle-mère. Selon Zill (1988), certains éléments mettent en évidence que les enfants vivant avec une belle-mère éprouvent plus de problèmes que ceux vivant avec un beau-père.

\section{Problèmes de comportement}

Les problèmes de comportement manifestés par les enfants vivant en famille recomposée ont aussi été fréquemment examinés par les chercheurs. Dans une étude longitudinale s'échelonnant sur six ans, Hetherington et al. (1985) ont examiné les effet de la séparation parentale et de la recomposition familiale sur les enfants. Les résultats font ressortir que le divorce a plus d'effets négatifs à long terme sur les garçons, alors que le remariage de la mère gardienne est associé à une augmentation des problèmes de comportement chez les filles et à une diminution de ces problèmes chez les garçons.

Cependant, si l'on compare ces enfants à ceux vivant en famille nucléaire, on constate que les garçons, tout comme les filles, manifestent davantage de problèmes de comportements (Bray, 1988).

On obtient sensiblement les mêmes résultats si I'on examine une dimension plus sévère des problèmes de comportement, soit la propension à commettre des actes déviants. En effet, une recherche menée par Steinberg (1987) auprès de 865 adolescents vivant soit en 
famille nucléaire, monoparentale ou recomposée n'a pas permis de confirmer l'hypothèse voulant que la présence d'un beau-parent puisse décourager l'adoption de comportements déviants comme $c^{\prime}$ est le cas quand un adolescent ou une adolescente vit avec ses deux parents biologiques. L'auteur conclut donc que les adolescents vivant en famille recomposée sont un groupe qui risque autant de s'engager dans des comportements déviants que le sont les adolescents vivant en famille monoparentale.

Notons que les résultats obtenus par Zill (1988) laissent aussi entendre que le degré de problèmes vécu par les enfants vivant en famille recomposée est comparable à celui des enfants de famille monoparentale et supérieur à ceux vivant en famille nucléaire. II a entre autres noté que les principales différences s'observaient sur le plan du comportement, de l'ajustement émotif et de l'utilisation de services de santé mentale.

Enfin, la présence de demi-frères ou de demi-sœurs serait notamment associée à un degré plus élevé de troubles comportementaux chez les enfants (Zill, 1988). Les travaux d'Ambert (1986) indiquent cependant, à ce sujet, que la qualité de la relation entre les enfants de deux familles différentes est plus positive lorsque ces derniers vivent ensemble de façon permanente. On ne peut cependant conclure qu'une meilleure qualité de relation entre les enfants venant de deux familles différentes ait nécessairement un impact positif sur le comportement des enfants.

\section{Contacts avec le parent non gardien}

Une des caractéristiques des enfants vivant en famille recomposée à la suite d'un divorce est qu'ils ont un parent avec qui ils ne vivent pas de façon régulière mais avec lequel ils ont des contacts plus ou moins fréquents. À ce sujet, une recherche menée par Knaub et Hanna (1984-b) a démontré que parmi leur échantillon, 47 \% des enfants vivant en famille recomposée ne souhaitaient jamais vivre avec leur parent non gardien, alors que $35 \%$ le souhaitaient quelquefois. Par ailleurs, les garçons semblaient plus portés que les filles à vouloir vivre avec leur parent non gardien, malgré le fait qu'ils percevaient plus positivement leurs relations avec leur beau-parent que les filles.

II apparaît, de plus, que les enfants ayant des contacts réguliers avec le parent non gardien éprouvent moins de problèmes que ceux ayant peu ou pas de contacts. Cette relation s'avère cependant fausse dans les familles recomposées comptant une belle-mère et, donc, une mère non gardienne. Ainsi, des contacts avec une mère non gardienne semblent plus critiques pour le bien-être de l'enfant que des contacts avec un père non gardien (Zill, 1988). De plus, on a constaté 
que de fréquentes visites chez la mère non gardienne sont associées à une relation moins positive dans la dyade fille-belle-mère (Clingempeel et Segal, 1986).

\section{Perception de la famille}

Une des premières études dans ce domaine a été menée par Duberman (1973) et a révélé que la relation beau-parent-enfant ainsi que la relation entre les enfants issus de deux familles différentes étaient jugées positives par les membres de la famille recomposée. II apparaît cependant que les enfants venant de famille divorcée (que les parents soient remariés ou pas) perçoivent leur famille comme moins bien adaptée que les enfants venant de famille nucléaire (Mitchell, 1983).

On constate par ailleurs qu'une exposition à la famille recomposée atténue les stéréotypes négatifs, particulièrement à l'endroit de la belle-mère (Fine, 1986). Cependant, une fois devenus adultes, les enfants élevés en famille recomposée perçoivent moins positivement leurs relations avec leur mère "non gardienne » et leur belle-mère comparativement aux enfants élevés en famille nucléaire. Encore une fois, aucune différence significative n'a été observée chez les personnes ayant été élevées par un beau-père ou par un père biologique (Fine et Sauer, 1988).

Knaub et Hanna (1984-b) ont constaté, parmi leur échantillon d'enfants vivant en famille recomposée, qu'une forte majorité d'entre eux considèrent que leur famille est un succès (45\%) ou un très grand succès $(25 \%)$. Cette étude a aussi permis de conclure que plus les enfants perçoivent leur famille comme adaptée à sa nouvelle situation, plus ils identifient de forces familiales en présence. Les résultats ne démontrent aucune différence dans la perception des garçons ou des filles. Cependant, les enfants plus jeunes semblent plus satisfaits, les plus âgés indiquant vivre plus de conflits.

On remarque donc que la perception qu'a un enfant de sa famille recomposée est associée à son âge. En effet, les recherches ont fait ressortir plusieurs enjeux particuliers lorsque la nouvelle famille compte des adolescents. Pink et Wampler (1985), dans une étude sur la qualité de la relation beau-parent-adolescent, ont constaté un bas niveau de cohésion et d'adaptation ainsi qu'une évaluation faible, en ce qui regarde la qualité de cette relation, telle qu'exprimée par les parents et les adolescents.

D'autres recherches sont arrivées à des résultats semblables. On remarque généralement peu de différences sur le plan de la perception qu'ont les filles et les garçons de leur beau-parent, mais la relation adolescente-beau-père apparaît plus distante sur le plan émotif 
que dans les autres dyades formées d'un adolescent et d'un beauparent (Clingempeel et al., 1984-a; Ganong et Coleman, 1987). Par ailleurs, les adolescents ne se sentent pas plus distants à l'égard d'un beau-père ou d'une belle-mère. Généralement, ils se considèrent modérément proches de leur beau-parent. On a aussi noté que le temps écoulé depuis la recomposition et la cause de la dissolution de la précédente famille ne semblent pas affecter la proximité entre les adolescents et les beaux-parents (Ganong et Coleman, 1987).

Une étude (Hobart, 1988) allant dans le même sens que celle de Brand et Clingempeel (1987) s'est intéressée à la perception qu'ont les parents et les beaux-parents de leurs relations avec leurs enfants, avec les enfants du conjoint et les enfants issus de la nouvelle union. De plus, Hobart a comparé cette perception avec celle de parents de famille nucléaire. Les résultats démontrent que la relation qu'entretiennent les parents avec les différentes catégories d'enfants varie. Les parents de famille nucléaire ou recomposée partagent sensiblement les mêmes perceptions à l'endroit des enfants issus de leur union, alors que cette perception varie grandement quand il s'agit des enfants nés d'une union antérieure. De plus, les parents semblent conscients de ces différences et les imputent au degré d'engagement de l'enfant dans la relation. Plus spécifiquement, on a noté que le plus grand facteur de prédiction de la perception différenciée des parents et des beaux-parents vis-à-vis des enfants est l'âge de ces derniers. Les adolescents étant plus provocateurs et cherchant plus à s'affirmer et à s'opposer, c'est tout naturellement que ce type de comportements sera mieux accepté par le parent naturel que par le beau-parent. Aussi, selon Hobart (1988), il faut s'attendre à ce que plus d'agents stressants soient associés aux adolescents lorsqu'une famille recomposée vient consulter.

Par ailleurs, le divorce ou le remariage ne semble pas affecter la perception qu'a l'enfant du soutien qui lui vient de sa mère (Amato, 1987). Pour plusieurs enfants, le soutien et l'amour qu'ils ont perdus de leur père biologique est remplacé, dans le temps, par celui du beau-père. De plus, et tout comme pour les enfants de famille monoparentale, les enfants de famille recomposée semblent avoir beaucoup de responsabilités. Cependant, même s'ils indiquent ne pas avoir plus d'autonomie que les enfants vivant en famille nucléaire, Amato (1987) affirme que ce dernier résultat fait apparaître des tensions au sujet du rôle des enfants dans les familles recomposées.

\section{Conception de soi des enfants dans les familles recomposées}

Partant du principe que la conception de soi des enfants est largement influencée par les caractéristiques et les attitudes des figures 
parentales qu'ils ont autour d'eux et que ce même concept est reconnu comme étant un bon indicateur de santé mentale (Kappes, 1980, cité dans Hutchinson, 1989), plusieurs chercheurs se sont intéressés à comparer la conception de soi des enfants selon le type de structure familiale dans laquelle ils grandissent.

Les recherches dans ce domaine ont démontré que les enfants de famille recomposée s'évaluaient quelque peu plus positivement que les enfants issus de familles monoparentales. De plus, ces enfants évaluent leur père plus positivement que leur mère (Parish et Dostal, 1980; Bray, 1988; Johnson et Hutchinson, 1989). Il n'y aurait donc pas de différences significatives dans l'image de soi des enfants selon la structure familiale dans laquelle ils grandissent. À la lumière de ces résultats, Johnson et Hutchinson (1989) concluent que :

Our society is inclined to view family structures other than the intact, nuclear family as potentially pathological. However, viewing the decline of the nuclear family as the cause of child pathology may be erroneous [...] It is possible and even probable that many children of divorce and those who became stepchildren obtain strengths and maturities associated with their experiences. (p. 137)

Cependant, même si les études n'ont pas permis de relever de différences significatives entre la conception de soi des enfants et la structure familiale, il est intéressant de noter que les enfants de famille nucléaire ont un peu plus tendance à se décrire comme étant "heureux", que les enfants de famille monoparentale ou recomposée. Les enfants de famille recomposée ont pour leur part un peu plus tendance à se décrire comme étant " courageux ", " équitables ", " honnêtes » et " avisés » (Bray, 1988).

La conception de soi des enfants semble influencée par la façon dont ils évaluent leurs parents. II apparaît toutefois que les enfants de famille recomposée ont tendance à avoir une conception d'euxmêmes qui correspond à la façon dont ils évaluent leur beau-père (Parish et Copeland, 1979).

Les résultats des travaux de Nunn et Parish (1987) laissent cependant apparaître que le temps passé à vivre avec un beau-parent influence cette association. De jeunes enfants auraient donc davantage tendance, même s'ils vivent en famille recomposée, à s'évaluer selon l'image qu'ils ont de leur parent naturel.

Bien que la structure familiale ne laisse pas apparaître de distinction dans la conception de soi des enfants, il semble que la perception du fonctionnement familial et le sexe du beau-parent aient davantage d'influence. Cette perception aurait notamment des conséquences sur le niveau d'estime de soi des adolescents (Pasley et Healow, 1988). 


\section{Impact de la qualité de la relation conjugale sur la relation beau-parent-enfants}

La raison d'être d'une famille recomposée est d'abord et avant tout le désir de deux adultes de vivre ensemble. Le couple est donc souvent considéré, avec raison, comme la pierre angulaire de la famille. De plus, on constate généralement chez les familles nucléaires qu'une relation conjugale positive a une influence positive sur les enfants. À partir de ce principe, quelques chercheurs ont voulu examiner le lien qui s'opère entre la qualité de la relation beau-parentenfants et la qualité de la relation du couple.

Afin d'y arriver, Brand et Clingempeel (1987) ont mené une étude auprès de familles recomposées comprenant un beau-père ou une belle-mère. Les résultats font ressortir que dans les familles avec belle-mère, une qualité de relation conjugale élevée est associée à une meilleure qualité de la relation entre la belle-mère et les garçons et une meilleure adaptation de ces derniers. La relation inverse a cependant été observée avec les filles. Du côté des familles comprenant un beau-père, la qualité de la relation avec les enfants des deux sexes et l'adaptation de ces derniers étaient reliées à la qualité de la relation conjugale.

La recherche d'Hetherington (1987) confirme par ailleurs qu'une relation inverse s'observe, lorsqu'il y a des filles dans la famille, entre la qualité de la relation conjugale et la qualité de la relation beauparent-enfants. En effet, l'augmentation de la qualité de la relation conjugale a été associée à une augmentation des conflits familiaux et des problèmes de comportements, particulièrement chez les filles. Ces résultats sont tout à fait en contradiction, selon Hetherington (1987), avec ce que l'on trouve généralement dans les études portant sur la famille nucléaire. Cette différence pourrait notamment s'expliquer, selon Brand et Clingempeel (1987), par le processus de socialisation des filles à qui l'on attribue plus facilement des rôles de parent et de prise en charge de la famille. Durant la phase de monoparentalité, les filles occuperaient une place de conjointe et apprécieraient cette position. L'arrivée d'une belle-mère soulèverait une certaine compétition. Les garçons étant plus portés à garder une position d'enfants, ils ne vivraient pas cette perte de rôle à la suite de la recomposition.

L'étude de Brand et al. (1988) permet aussi d'expliquer la relation inverse que l'on observe entre la qualité de la relation conjugale et de la relation beau-parent-enfants. Cette étude, menée auprès de 40 familles recomposées avec beau-père et 22 familles avec belle-mère, a démontré que la relation belle-mère-filles est plus problématique que la relation belle-mère-garçons. Pour les filles, on a observé une rela- 
tion moins positive avec la belle-mère et un niveau d'ajustement psychologique plus bas lorsque la relation conjugale est évaluée comme positive. De plus, de fréquentes visites de la mère non gardienne ont été associées à une moins bonne relation bellè-mère-filles (selon la perception des belles-filles). Finalement, une relation belle-mèrefilles, qualifiée de positive, a été associée à un meilleur ajustement psychologique des belles-filles. À partir d'autres travaux empiriques, Brand et al. (1988) croient que l'on peut expliquer la différence entre l'ajustement des garçons et des filles par le fait que dans les familles recomposées comprenant une belle-mère, les pères encouragent et soutiennent l'indépendance chez leurs garçons, mais pas chez leurs filles. Dans les faits, on renforcerait chez les filles la non-indépendance et le maintien de relations enchevêtrées avec le père (Greig, 1985, cité dans Brand et al., 1988). Encore une fois dans l'étude de Brand et al. (1988), le sexe de l'enfant et le type de famille recomposée ont été vus comme des facteurs importants dans l'étude des familles recomposées.

\section{Conclusion}

Que conclure de l'ensemble des recherches examinées ici, si ce n'est qu'il faudra attendre quelques années avant que ne se dégagent de véritables certitudes. Comme le lecteur a pu le constater, il est fréquent que les résultats obtenus dans une étude soient contredits dans une autre pour faire place à de nouvelles explications. Néanmoins, il se dégage certains faits qui, à défaut d'être complètement expliqués, fournissent au chercheur et à l'intervenant certaines pistes de réflexion.

Tout d'abord, on constate que les familles recomposées sont structurellement différentes des autres modèles familiaux et qu'elles doivent de ce fait fonctionner différemment. De cette première constatation en découle une autre, soit l'absence de normes institutionnalisées pouvant servir de guide ou de modèle aux familles recomposées.

Par ailleurs, les questions de ce genre semblent particulièrement importantes dans l'étude des familles recomposées. Premièrement, la question de l'influence du sexe de l'enfant sur différents thèmes touchant la recomposition familiale ne fait pas consensus. Ainsi, selon Clingempeel et al. (1984-a) et Hetherington (1987), la relation qu'entretiennent les filles avec leur beau-parent est plus problématique que pour les garçons. À l'inverse pour Mitchell (1983), les filles s'adapteraient mieux que les garçons à une situation de recomposition. Bray (1988) considère pour sa part que les garçons profitent davantage de la 
recomposition familiale que les filles même s'ils ont plus de problèmes de comportement. Finalement, Kurdek et Sinclair (1988) ont indiqué que le sexe de l'enfant ne semble pas interférer dans son ajustement. II faudra donc davantage explorer cette question afin de vraiment saisir l'influence du sexe de l'enfant sur la recomposition.

Toujours sur la question du genre, nous avons été frappés par l'ensemble des résultats de recherche identifiant, à un niveau ou à un autre, l'importance du sexe de l'enfant ou du beau-parent dans l'ajustement à une situation de recomposition. Si nous voulions brosser un portrait type des familles recomposées risquant le plus de vivre des problèmes, nous dirions qu'il s'agit de celles impliquant une bellemère, la ou les filles du conjoint et la présence fréquente d'une mère non gardienne. En effet, le rôle de beau-père semble moins critique que celui de belle-mère, les garçons profitent plus de la recomposition que les filles, ces dernières s'épanouissent plus à l'intérieur d'une famille recomposée où le couple vit des difficultés de relations, et les relations belle-mère-filles semblent mises en péril par le maintien des contacts avec le parent non gardien, spécialement quand ce parent est une femme. Les difficultés vécues par les femmes dans les familles recomposées nous portent à croire que la recherche dans ce domaine aurait intérêt à examiner plus à fond le processus de socialisation des filles, de même que la question des rôles attribués aux hommes et aux femmes au sein de la famille.

Finalement, nous croyons que chercheurs comme intervenants devront se méfier du danger de stigmatiser la famille recomposée. S'il est vrai qu'elle est différente et qu'elle nécessite que nos interventions tiennent compte de cette différence, elle n'en demeure pas moins une structure familiale ayant les ressources et la capacité de répondre aux besoins de ses membres. Nous ne pouvons toutefois nier que cette nouvelle structure familiale vit des défis particuliers. Aussi croyons-nous que les recherches futures devront tenter de mieux comprendre ce qui facilite l'adaptation à la vie en famille recomposée plutôt que de trop souvent chercher à déterminer ce qu'elle risque d'offrir en moins à ses membres en comparaison de la famille nucléaire. Nous croyons qu'une telle perspective de recherche sera plus profitable et permettra d'assurer un peu plus de stabilité aux adultes et aux enfants qui croient, une "deuxième fois ", en la famille.

\section{Notes}

\footnotetext{
${ }^{1}$ Une partie de cette recension des écrits a été faite dans le cadre du mémoire de maîtrise de l'auteure.

${ }^{2}$ Fédération des CLSC du Québec, 1989.
} 
${ }^{3}$ Il faut noter que la question de l'abus sexuel des enfants dans les familles recomposées retient de plus en plus l'attention des chercheurs. Cependant, en raison de la spécificité de ce thème, nous avons choisi de ne pas l'aborder à l'intérieur de cette recension.

${ }^{4}$ Le repérage des articles ayant servi à développer cette recension s'est fait à l'aide des bases de données informatiques Psycinfo, Psyclit et Sociofile. Les principaux mots clés utilisés ont été : remarriage, stepfamily, reconstitution, blended families et stepchildren. Le lecteur notera, toutefois, que cette revue des écrits n'a aucune prétention d'exhaustivité. Plus modestement, nous avons cherché à faire état des thèmes ayant le plus retenu l'attention des chercheurs dans ce domaine.

${ }^{5}$ Bien que le terme "famille nucléaire » ne soit pas parfait, nous le préférons à famille traditionnelle » ou "famille intacte ». Aussi, tout au long de ce texte, " famille nucléaire » désignera la famille composée des deux parents et des enfants issus de cette union.

${ }^{6}$ La différence entre les $14 \%$ et les $9,4 \%$ s'explique par le fait qu'un certain nombre d'enfants vivent en famille recomposée, mais sont issus de cette famille. Comme tel, ces enfants vivent avec leur deux parents naturels, de même qu'en présence de demi-frères ou demi-sœurs.

7 Exception faite d'une personne qui en serait à sa toute première union.

${ }^{8}$ À ce sujet, il est intéressant de noter que selon une étude menée par Furstenberg et al. (1983), des contacts fréquents (c'est-à-dire au moins une fois par semaine) entre les enfants et le parent non gardien sont apparus dans seulement $17 \%$ des familles séparées depuis cinq ans.

\section{Références bibliographiques}

AlBreCHT, Stan L. (1979). "Correlates of marital happiness among the remarried ", Journal of Marriage and the Family, vol. $41:$ 857-867.

AlbreCHT, Stan L. (1983). Divorce and remarriage: Problems, adaptations and adjustments. Wesport : Greenwood Press, p. 141-156.

AMATO, Paul R. (1987). "Family processes in one-parent, stepparent, and intact families : The child's point of view ", Journal of Marriage and the Family, vol. 49 : 327-337.

AMBERT, Anne-Marie (1983). "Separated women and remarriage behavior : A comparison of financially secure women and financially insecure women ", Journal of Divorce, vol. 6 : 43-54.

AMBERT, Anne-Marie (1986). "Being a stepparent living and visiting stepchildren ", Journal of Marriage and the Family, vol. 48 : 795-804.

ANDERSON, Judith Zucker et Geoffroy D. WHITE (1986). " An empirical investigation of interaction and relationship patterns in functional and dysfunctional nuclear families and stepfamilies ", Family Process, vol. 25 : 407-422.

AtweLL, A.W., U.S. MOore et C.S. Nowell (1982). "The role of stepparents in child custody disputes ", Bulletin of The American Academy of Psychiatry \& the Law, vol. $10: 211-217$.

BACHRACH, Christine A. (1983). "Children in families : Characteristics of biological, step and adopted children ", Journal of Marriage and the Family, vol. $45: 171-179$. 
BAHR, Stephen J. (1979). "The effects of welfare on marital stability and remarriage ", Journal of Marriage and the Family, vol. 41 : 553-560.

BoIsGelot, Christianne (1989). T'es pas ma mère! Paris : éd. Olivier Orban.

BOWERMAN, C.E. et D.P. IRISH (1962). "Some relationships of stepchildren to their parents ", Marriage and Family Living, vol. 24 : 113-121.

BRAND, Eulalee et W. Glenn Clingempeel (1987). "Interdependencies of marital and stepparent-stepchild relationships ans children's psychological adjustment : Research findings and clinical implications", Family Relations, vol. $36: 140-145$.

Brand, Eulalee, W. Glenn Clingempeel et Kathryn Bowen-Woodward (1988). "Family Relationships and children's psychological adjustment in stepmother and stepfather families ", dans Impact of Divorce, Single Parenting and Stepparenting on Children, Hetherington E. Mavis \& Arasteh Josephine D., eds, Lawrence Erlbaum Associates, pub., New Jersey, 389 p.

BRAY, James H. (1988). "Children's development during early remarriage", dans Impact of Divorce, Single Parenting and Stepparenting on Children. E. Mavis Hetherington et Josephine D. Arasteh (éd.), New Jersey: Lawrence Erlbaum Associates, publ.

Brody, Gene H., Eileen Neubaum et Rex Forehand (1988). "Serial marriage : An heuristic analysis of an emerging family form ", Psychological Bulletin, vol. $103: 211-222$.

Buehler, C., M.J. Hogan, B. Robinson et R.J. Levy (1986). « Remarriage following divorce; stressors and well-being of custodial and noncustodial parents ", Journal of Family Issues, vol. 7 : 405-420.

BURCH, Thomas K. (1985). Enquête sur la famille - conclusions préliminaires, Statistique Canada, ministère des Approvisionnements et Services Canada, 99-955.

Burgoyne, Jacqueline et David Clark (1982). "Reconstituted Families", chap. 14, dans Rapoport et al. (éd.) Families in Britain. Londres : Routledge and Kegan.

CAPALDI, Frederick et Barbara MCRAE (1979). Stepfamilies : A cooperative responsability. New York: New Viewpoints/Vision Books.

CHAPMAN, Michael (1977). "Father absence, stepfathers, and the cognitive performance of college students ", Child Development, vol. 48 : 1155-1158.

CherLiN, A. (1978). "Remarriage as an incomplete institution ", American Journal of Sociology, vol. 84 : 634-650.

CHERLIN, A. et J. MCCARTHY (1985). "Remarried couple household : data from the June 1980 current population survey », Journal of Marriage and the Family, vol. $47: 23-30$.

Clingempeel, Glenn W. (1981). "Quasi-kin relationships and marital quality in stepfather families ", Journal of Personality and Social Psychology, vol. 41 : 390-901.

Clingempeel, Glenn W. et Eulalee Brand (1985). "Quasi-kin relationships, structural complexity, and marital quality in stepfamilies : a replication, extension, and clinical implications ", Family Relations, vol. 34 : 401-409.

Clingempeel, Glenn W., Eulalee Brand et Richard Ievoli (1984-a). "Stepparentstepchild relationships in stepmother and stepfather families : A multimethod study ", Family Relations, vol. 33 : 465-473.

Clingempeel, Glenn W., Eulalee Brand et Richard Ievol (1984-b). "Structural complexity and the quality of stepfather-stepchild relationships ", Family Process, vol. 23 : 547-560. 
Clingempeel, Glenn W. et Sion Segal (1986). "Stepparent-stepchild relationships and the psychological adjustment of children in stepmother and stepfather families", Child Development, vol. 57 : 474-484.

Coleman, Marylin et Lawrence H. GANONG (1984). " The effects of remarriage on children : A review of the empirical literature », Family Relations, vol. 33 : 389-406.

COleman, Marylin et Lawrence H. GANONG (1986). « A comparison of clinical and empirical literature on children in stepfamilies ", Journal of Marriage and the Family, vol. $48: 309-318$.

COleman, Marylin et Lawrence H. GANONG (1987). " The cultural stereotyping of stepfamilies ", dans Remarriage \& Stepparenting - Current Research \& Theory. New York : Pasley \& Ihinger-Tallman, (éd.), Guilford Press.

CROSBIE-BIRNETT, Margaret (1984). "The centrality of the step relationship : a challenge to family theory and practice», Family Relations, vol. 33 : 459-463.

DEMARIS, Alfred (1984). "A comparison of remarriages with first marriages on satisfaction in marriage and its relationship to prior cohabitation », Family Relations, vol. 33 : 443-449.

DUBERMAN, Lucile (1973). "Step-kin relationships », Journal of Marriage and the Family, vol. 35 : 283-292.

Duberman, Lucile (1975). The Reconstituted Family. Chicago : Nelson-Hall.

DUKES, Richard L. (1989). "The Cinderella myth : Negative evaluations of stepparents ", Sociology and Social Research, vol. 73 : 67-72.

FAST, I. et A.C. CAIN (1966). "The stepparent role : potential for disturbances in family functionning ", American Journal of Orthopsychiatry, vol. 36 : 485-491.

FERRI, Elsa (1984). Stepchildren : a National Study, a Report from the National Child Development Study. Londres : NFER-Nelson.

FINE, Mark A. (1986). "Perceptions of stepparents : Variation in stereotypes as a function of current family structure ", Journal of Marriage and the Family, vol. 48 : 537-543.

FISHERMAN, Barbara et Bernice HAMEL (1981). "From nuclear to stepfamily ideology - A stressful change ", Alternative Lifestyles, vol. 4 : 181-204.

FURSTENBERG, F.F. Jr. (1980). "Reflections on remarriage", Journal of Family Issues, special issues on remarriage, vol. $1: 443-453$.

FURSTENBERG, F.F. Jr. et Graham B. SPANIER (1984-a). " The risk of dissolution in remarriage; an examination of Cherlin hypothesis of incomplete institutionalization ", Family Relations, vol. 33 : 433-441.

FurstenberG, F.F. Jr. et Graham B. SPANIER (1984-b). Recycling the Family, Remarriage after Divorce, Beverly Hills, Ca. : Sage Publications.

FurstenberG, F.F. Jr., C. WINQUist-Nord, J.L. Peterson et N. Zill (1983). "The lifecourse of children of divorce : Marital disruption and parental contact ", American Sociological Review, vol. 48 : 656-668.

Galvin, Kathleen M. et Bernard J. Brommel (1986). "Communication within stepfamily system ", dans Family Communication, Cohesion and Change ( $\mathrm{e}^{\mathrm{e}}$ édit.), III. Glenview : Scott, Foresman and Co.

GANONG, Lawrence H. et Marilyn M. COLEMAN (1984). " Effect of family structure on family attitudes and expectations ", Family Relations, vol. 33 : 425-432.

GanonG, Lawrence H. et Marilyn M. Coleman (1987). "Stepchildren's perceptions of their parents ", Journal of Genetic Psychology, vol. 148 : 5-17. 
Germain, Diane (1986). "La famille reconstituée : Le deuil de l'idéal », dans Pierre Gauthier, Les nouvelles familles. Québec : Éd. Saint-Martin.

GLENN, Norval et Charles N. WEAVER (1977). "The marital happiness of remarried divorced persons ", Journal of Marriage and Family Therapy, vol. 39 : 331-337.

Gııck, Paul C. (1989). "Remarried families, stepfamilies, and stepchildren : A brief demographic profile ", Family Relations, vol. 38 : 24-27.

Glick, Paul C. et Sung-Ling LIN (1986). "Recent changes in divorce and remarriage ", Journal of Marriage and the Family, vol. 48 : 737-747.

GoldSTEIN, H.S. (1974). "Reconstituted families: The second marriage and its children », Psychiatric Quarterly, vol. 48 : 433-440.

GOLMEIER, John (1980). "Intervention in the continuum from divorce to family reconstitution ", Social Casework, vol. $61: 39-47$.

Guisinger, S., Philip A. Cowan et David Schuldberg (1989). "Changing parent and spouse relations in the first years of remarriage of divorced fathers", Journal of Marriage and the Family, vol. 51 : 445-456.

HALPERIN, S.M. et T.A. SMITH (1983). "Differences in stepchildren's perceptions of their stepfathers and natural fathers : implications for family therapy ", Journal of Divorce, vol. 7 : 19-30.

HanNa, Sharon L. et Patricia KaIn KNAUb (1981). "Cohabitation before remarriage : its relationship to family strengths", Alternative Lifestyles, vol. 4 : 507-522.

Hetherington, Mavis E., Jeffrey D. Arnett et Ann E. Hollier (1988). « Adjustment of parents and children to Remarriage ", chap. 3, dans Children of Divorce, Wolchik Sharlene A. \& Karoly Paul. New York : Gardner Press inc.

Hetherington, Mavis E. (1987). "Family Relations six years after divorce ", dans Remarriage \& Stepparenting - Current Research \& Theory. Pasley et Ihinger-Tallman (éd.), New York : Guilford Press.

Hetherington, Mavis E. et Josephine D. Arasteh (1988). Impact of Divorce, Single Parenting and Stepparenting on Children. New Jersey : Lawrence Erlbaum Associates, publ.

Hetherington, Mavis E., Martha Cox et Roger Cox (1985). " Long-term effects of divorce and remarriage on the adjustment of children ", Journal of the American Academy of Child Psychiatry, vol. 24 : 518-530.

HOBART, Charles W. (1988). "Perception of parent-child relationships in first married and remarried families ", Family Relations, vol. 37 : 175-182.

HutChINSON, Roger L., William E. Valutis, Deborah T. Brown et Steven J. WHITE (1989). " The effects of family structure on institutionalized children's selfconcepts ", Adolescence, vol. $24: 303-310$.

JACOBSON, Doris S. (1979). "Stepfamilies: Myths and realities", Social Work, vol. 24 : 202-207.

JaCOBSON, Doris S. (1980). "Stepfamilies", Children Today, vol. 9 : 2-6.

JOHNSON, Melanie K. et Roger L. HUTCHINSON (1989). " Effects of family structure on children's self-concepts", Journal of Divorce, vol. $12: 129-138$.

KENT, Marilyn O. (1980). "Remarriage : A family system perspective », Social Casework, vol. 61 : 146-153.

KESHET, H.F. et K.M. ROSENTHAL (1978). "Fathering after marital separation ", Social Work, vol. $23: 11-18$. 
KNAUB KaIN, Patricia et Sharon L. HANNA (1984-a). "Strengths of remarried families ", Journal of Divorce, vol. 7, $n^{\circ} 3: 41-55$.

KNaUb KaIN, Patricia et Sharon L. Hanna (1984-b). "Children of remarriage : perceptions of family strengths » Journal of Divorce, vol. 7, $n^{\circ} 4: 73-90$.

KOMPARA, Diane Reinhart (1980). " Difficulty in the socialization process of stepparenting ", Family Relations, vol. 29 : 69-73.

KURDEK, Lawrence A. et Ronald J. SINCLAIR (1988). "Adjustment of young adolescents in two-parent nuclear, stepfather, and mother-custody families ", Journal of Consulting and Clinical Psychology, vol. 56 : 91-96.

Lewis, Robert A. et Marvin B. Sussman (1985). "Men's changing roles in the family ", numéro spécial de Marriage and Family Review, vol. 9, nºs 3-4. New York: Haworth Press.

MEAD, Margaret (1970). "Anomalies in American post-divorce relationships ", dans Divorce and After Divorce, P. Bohannan (ed.). New York : Doubleday.

MESSINGER, L. (1976). " Remarriage between divorced people with children from previous marriage : a proposal for preparation for remarriage ", Journal of Mariage and Family Counseling, vol. 2 : 193-200.

Messinger, L., K.N. Walker et S.J. Freeman (1978). "Preparation for remarriage following divorce : the use of group techniques", American Journal of Orthopsychiatry, vol. $48: 263-272$.

MiTCHELL, Kristen (1983). "The price tag of responsability : a comparison of divorced and remarried mothers", Journal of Divorce, vol. $6: 33-42$.

MORRISSON, K. et A. THOMPSON-GuPPY (1986). "Cinderella's stepmother syndrome ", Canadian Journal of Psychiatry, vol. 30 : 521-529.

Nelson, Margaret et Gordon K. Nelson (1982). "Problems of equity in the reconstituted family: A social exchange analysis », Family Relations, vol. $31: 223-231$.

NOLAN, Jeanne, Marilyn COLEMAN et Lawrence GANONG (1984). "The presentation of stepfamilies in marriage and family textbooks ", Family Relations, vol. 33 : 559-566.

NUNN, Gerald D. et Thomas S. PARISH (1987). "An investigation of the relationships between children's self-concepts and evaluations of parent figures : Do they vary as a function of family structure? ", The Journal of Psychology, vol. 12 : 563-566.

NunN, Gerald D., Thomas S. PARISH et Ralph J. WorTHING (1983). «Perceptions of personal and familial adjustment by children from intact, single-parent and reconstituted families", Psychology in the Schools, vol. 20 : 166-174.

PAPERNOW, Patricia L. (1984). "The stepfamily cycle : An experiential model of stepfamily development ", Family Relations, vol. 33 : 355-363.

PARISH, Thomas S. et Terry F. COPELAND (1979). " The relationship between selfconcepts and evaluations of parents and stepfathers ", Journal of Psychology, vol. $101: 135-138$.

PARISH, Thomas S. et Judy W. Dostal (1980). " Evaluations of self and parent figures by children from intact, divorces and reconstituted families ", Journal of Youth and Adolescence, vol. 9 : 347-351.

Pasley, B. Kay et Cathy L. Healow (1988). "Adolescent self-esteem : A focus on children in stepfamilies", dans Impact of Divorce, Single Parenting and Stepparenting on Children, Mavis E. Hetherington \& Josephine D. Arasteh, eds., Lawrence Erlbaum Associates, pub., New Jersey, 389 p. 
Pasley, B. Kay et Marilyn Ihinger-Tallman (1985). "Portraits of stepfamily life in popular literature : 1940-1980 », Family Relations, vol. 34 : 527-534.

PASLeY, B. Kay et Marilyn IHINGer-TAlLmAN (1989). "Boundary ambiguity : Does ambiguity differentiate degree of marital adjustment and interaction ? ", Family Relations, vol. 38 : 46-52.

Pasley, B. Kay, Marilyn Ihinger-Tallman et Cathy Coleman (1984). "Consensus styles among happy and unhappy remarried couples ", Family Relations, vol. 33 : 451-457.

PERKINS, Terry F. et James P. KAHAN (1979). " An empirical comparison of naturalfather and stepfather family systems ", Family process, vol. 18, $\mathrm{n}^{\circ} 2$ : 175-183.

Peron, Yves, Évelyne Lapierre-Adamcyk et Denis Morissette (1987). "Le changement familial : Aspects démographiques", Recherches sociographiques, vol. 28, $\mathrm{n}^{\text {os }} 2-3$ : 317-339.

PINK, Jo Ellen Theresa et Karen SMITH WAMPLER (1985). "Problems areas in stepfamilies : cohesion, adaptability, and the stepfather-adolescent relationship », Family Relations, vol. 34, n 3 : 327-335.

ROBERTS, Thomas W. et Sharon J. PRICE (1985). "A system analysis of the remarriage process : Implications for the clinician", Journal of Divorce, vol. $9, \mathrm{n}^{\circ} 2: 1-25$.

RoberTS, Thomas W. et Sharon J. PrICE (1987). "Instant families : Divorced mothers marry never-married men $"$, Journal of Divorce, vol. 11, $\mathrm{n}^{\circ} 1$ : 71-92.

ROBERTS, Thomas W. et Sharon J. PRICE (1989). "Adjustment in remarriage : Communication, cohesion, marital and parental roles ", Journal of Divorce, vol. 13, $\mathrm{n}^{\circ} 1:$ 17-43.

ROBINSON, Bryan E. (1984). "The contemporary American stepfather », Family Relations, vol. 33, $n^{\circ} 3: 381-388$

Roosevelt, R. et J. Lofas (1976). Living in Step. New York : Stein and Day.

ROSENBORG, B. Elinor et Fady HAJAL (1985). "Stepsibling relationships in remarried families", Social Casework, vol. 66 : 287-292.

RUSSEL, Diana E.H. (1984). "The prevalence and seriousness of incestuous abuse : stepfathers vs. biological fathers", Child Abuse and Neglect, vol. $8: 15-22$.

SAGer, Clifford J., Elisabeth Walker, Steer Hollis Brown et Helen Crovan (1981). "Improving the functionning of the remarried family system », The Journal of Marital and Family Therapy, vol. 7, $\mathrm{n}^{\circ} 1:$ 3-13.

SAINT-JACQUES, Marie-Christine (en cours). Étude des facteurs de prédiction de la tension de rôle chez les familles recomposées, mémoire de maîtrise, École de service social, Université Laval.

SANTROCK, John W., Richard WARSHAK, Cheryl LindBeRGH et Larry MeAdows (1982). "Children's and parents' observed social behavior in stepfather families", Child Development, vol. 53 : 472-480.

SAUer, Lawrence E. et Mark A. Fine (1988). "Parent-child relationships in stepparent families", Journal of Family Psychology, vol. 1 : 435-451.

SPANIER, G.B. et P.C. Glick (1981). "Marital instability in the United States : some correlates and recent changes", Family Relations, vol. 30, n $3: 329-338$.

Statistique Canada (1988). Le mariage et le divorce - Examen de la situation au Canada. Ministère des Approvisionnements et Services Canada, 89-517F. 
Statistique Canada (1989). La famille au Canada - Faits saillants choisis. Ministère des Approvisionnements et Services Canada, 89-509.

STEINBERG, Laurence (1987). "Single parents, stepparents, and the susceptibility of adolescents to antisocial peer pressure ", Child Development, vol. 58 : 269-275.

Stollman, Wilma (1985). Familles reconstituées... avec succès ! Service à la famille Canada, $94 \mathrm{p}$.

THÉRY, Irène (1987). "Remariage et familles composées : des évidences aux incertitudes", L'année sociologique, $\mathrm{n}^{\circ} 37: 119-152$.

TROPF, D. Walter (1984). " An exploratory examination of the effect of remarriage on child support, and personnal contacts ", Journal of Divorce, vol. 7 : 57-73.

TROST, Jan E. (1984). "Remarriage in Sweden ", Family Relations, vol. 33, n 3 : 475-481.

VISHER, Emily B. et John S. VISHER (1978). "Common problems of stepparents and their spouses ", American Journal of Orthopsychiatry, vol. 48, $\mathrm{n}^{\circ} 2: 252-262$.

VISHER, Emily B. et John S. VISHER (1979). Stepfamilies : a Guide to Working with Stepparents and Stepchildren. Secaurus, N.J. : The Citated Press, 280 p.

VISHER, Emily B. et John S. VISHER (1989). "Parenting coalitions after remarriage : Dynamics and therapeutic guidelines ", Family Relations, vol. 38, n ${ }^{\circ}$ 1: 6570.

VISHER, Emily B. et John S. VISHER (1990). « Dynamics of successful stepfamilies ", Journal of Divorce \& Remarriage, vol. 14, $\mathrm{n}^{\circ} 1: 3-12$.

WALKER, Glynnis (1984). Second Wife, Second Best ? Managing your Marriage as a Second Wife, Toronto : Doubleday Canada Ltd.

WALKER, Kenneth N. et Lillian MESSINGER (1979). "Remarriage after divorce : dissolution and reconstruction of family boundaries", Family Process, vol. $18, \mathrm{n}^{\circ} 2: 185-192$.

WALTERS, James et Nick STINNETT (1971). «Parent-child relationships : A decade of research ", Journal of Marriage and the Family, vol. 33, n $1: 70-111$.

WHITE, Lynn K. (1979). "Sex differentials in the effects of remarriage on global happiness ", Journal of Marriage and the Family, vol. 41, $n^{\circ} 4: 869-876$.

WHITE, Lynn K. (1983). " Determinants of spousal interaction : Marital structure and marital happiness", Journal of Marriage and the Family, vol. 45 : 511-519.

WHITE, Lynn K. et Alan BоOTH (1985). "The quality and stability of remarriages : The role of stepchildren ", American Sociological Review, vol. 50 : 689-698.

WiLson, Kenneth, Louis A. ZURCHER, Diana Claire MCADAMS et Russel L. CURTIS (1975). "Stepfathers and stepchildren : An exploratory analysis from two national surveys ", Journal of Marriage and the Family, vol. 37, n 3 : 526-536.

ZILL, Nicholas (1988). "Behavior, achievement, and health problems among children in stepfamilies : Findings from a national Survey of child health ", dans Impact of Divorce, Single Parenting and Stepparenting on Children, Mavis E. Hetherington et Josephine D. Arasteh (eds.), New Jersey : Lawrence Erlbaum Associates, publ. 